\title{
Research on the construction of supply chain network based on fixed spread risk
}

\author{
Lei Wen, Yachao Shi, Mingfang Guo \\ Department of Economics and Management, North China Electric Power University, Baoding 071003, China \\ Email address: \\ ncepuwxzj@163.com (Lei Wen), syc_0319@163.com(Yaochao Shi), mingfang_1100@126.com (Mingfang Guo)
}

To cite this article:

Lei Wen, Yachao Shi, Mingfang Guo. Research on the Construction of Supply Chain Network Based on Fixed Spread Risk. Science Journal of Applied Mathematics and Statistics. Vol. 1, No. 5, 2013, pp. 50-53. doi: 10.11648/j.sjams.20130105.13

\begin{abstract}
Supply chain is an integrated network where suppliers, sellers and customers are highly interconnected through business activities. The basic character of supply chain is complexity, dynamic and growth. In this paper, based on the complex network theory, we build a supply chain network model based on gradual risk. The dynamic growth mechanism of the supply chain network is based on the local world evolving model. The new node adds the local world entering with a certain probability. Combined with supply chain, the node, the edge and the weighted and the network are being redefined. The process of how new added node assign risk to old nodes is also introduced
\end{abstract}

Keywords: Supply Chain, Complex Networks, Risk Model, Generation Process

\section{Introduction}

Supply chain is a value network which is used to describe activity of each entity and their mutual relations. Or to be more precise, it described the process of businesses' flows, such as material, information and financial, from upstream enterprise to downstream enterprise [1-4]. Due to today's uncertain and turbulent changeable environment, supply chain enterprises today face various factors of uncertainties, which causing the enterprises can not work well. How to treat the influence of supply chain vulnerability on supply chain enterprises and how to take appropriate measures and subsequently control them has become an issue of significance for many companies [5].

Complex network is a useful tool to analyze the impact of risk spread on supply chain [6-10]. There has been increased attention focused on supply chain risk from various directions. Fabrizio Natalea et al [11] build a movement network to simulate epidemic spread in supply chain. Monique A. van der Gaaga Fred Vosa et al. [12] design a detailed stochastic state transition model and analyze its characteristics. Kathrin Buttnera et al [13] present a static poultry trade supply chain network to analyze its statistic characteristics. In this paper, we mainly study on construction of supply chain risk network based on complex network theory.

The rest of this paper is organized as follows: In the next section, we outline the risk spread model and put forward the definition of node, edge and weight. In section 3, we describe the process of supply chain risk network modeling, including the Selection of Local World, the process of the joining a new node and distributing risk value. In Section 4, we draw some concluding remarks.

\section{Risk Network Model}

The supply chain's contact structure may be visualized in terms of a network or graph. Each of the nodes in the network represents an enterprise which consists of supply, seller and customer. An edge is placed between any pair of enterprise who establishes relationship with each other. The resulting network topology is summarized by the symmetric adjacency matrix $A$ who elements $\mathrm{a}_{\mathrm{ij}}=0$ if there is the absence of such a contact, while $\mathrm{a}_{\mathrm{ij}}=1$ or $\mathrm{a}_{\mathrm{ij}}=-1$ if there is a contact between the node enterprise $i$ and $j$, which from upstream entity to downstream entity or contrary direction.

\subsection{Definition of Node}

The supply chain risk node is defined as vi $=(I D$, Category, Level, Risk $)$, where ID standards for the number of nodes in the supply chain, which is used to distinguish the different members of the enterprise; Category is a classification identifier of the node; The level of node shows the business order in supply chain; 
risk represents all kinds of risk that exists in supply chain risk network.

The node enterprises in supply chain network are divided into suppliers, sellers and customers in accordance with their business in the supply chain. The functions of the supplier to provide raw materials and finished products; the functions of the sellers is the procurement of goods and finished products to its upstream suppliers or vendors sold its downstream enterprises; the functions of the customer is the procurement of goods to the seller.

\subsection{Definition of Edge}

The edge expresses business relationship between business entities $\mathrm{v}_{\mathrm{i}}, \mathrm{v}_{\mathrm{j}}$ in the supply chain. There will be an edge between node $\mathrm{V}_{\mathrm{i}}, \mathrm{V}_{\mathrm{j}}$ if the business between business entities $\mathrm{V}_{\mathrm{i}}, \mathrm{V}_{\mathrm{j}}$ which express adjacent and downstream enterprises is related, or there is no. The edge denoted by $\mathrm{e}=\left(\mathrm{v}_{\mathrm{i}}, \mathrm{v}_{\mathrm{j}}\right)$, $\mathrm{e}$ is called the associated edge of $\operatorname{nodes} \mathrm{v}_{\mathrm{i}}, \mathrm{v}_{\mathrm{j}}$. Call $\mathrm{v}_{\mathrm{i}}, \mathrm{v}_{\mathrm{j}}$ as the end ofe.

\subsection{Definition of Weight}

In the supply chain, the weight $\mathrm{W}_{\mathrm{ij}}$ expresses logistics quantity between node $\mathrm{V}_{\mathrm{i}}$ and node $\mathrm{V}_{\mathrm{j}}$. The weight can be distinguished dissimilarity weight and similarity weight. And the dissimilarity weight is similar to the traditional distance, the greater the value, the bigger the distance and the more distant the relationship between two points. Similarity weight is conversely, the greater weight said the more intimate relationship, the smaller the distance between two points. So this paper uses the dissimilarity weight. And the direction of edge in the supply chain expresses the order of business between the associated enterprises; the starting node level must be less than the end nodes. That is, the level of the business entities $V_{i}$ should be less than the level of business entities $\mathrm{v}_{\mathrm{j}}$. It is to ensure that the edge direction and supply chain logistics volume in the same direction. So the $\mathrm{W}_{\mathrm{ij}}$ expresses the weight from node $\mathrm{V}_{\mathrm{i}}$ to node $\mathrm{V}_{\mathrm{j}}$. It is the incoming edge weight for $\mathrm{v}_{\mathrm{i}}$, but outgoing edge weight for $\mathrm{v}_{\mathrm{j}}$, and $\mathrm{w}_{\mathrm{ij}}=\mathrm{w}_{\mathrm{ji}}$.

\subsection{The Supply Chain Risk}

The network conceptual framework has been very useful for the description and analysis of the risk of transmission. Now, we will present the basic definitions of the components of risk network:

i) Risk source: the source of risk transmission is defined as risk source. The origin of risk by classification can be divided into external risks and internal risk. The risks caused by market fluctuations or government policy is often referred to as external risks; internal risks is any loss events that caused by internal operations.

ii) Risk nodes: in supply chain risk network, every enterprise can be called as risk nodes. Classify them by conduction target, risk nodes can be divided into risk object and risk subject. The risk nodes that transmit risk are referred to as risk object. Relatively, the risk nodes that infected risk are referred to as risk object. With the difference of risk source, any nodes can become risk object, as long as it is an originator. The same condition applies to risk subject.

iii) Risk carriers: in the process of conduction, risk at its essence is an intangible item that transmitted associated risk nodes through "the medium" or "the bridge", and these "media" or "bridges" are the risk conduction carriers. The risk conduction carrier has five characteristics: objectivity, multiplicity, load bearing, conductivity and orderliness. For different properties of risk carriers and risk source attribute, risks have had transmitted dependent on different medium.

iv) Transmission pathways: it can be defined as the logical distance of risk transmitted from one node to another. For different conduction pathways, the modes of risk conduction are different. In this network, transmission pathways started from suppliers, through sellers and finally reaching customers. Risk will attach to all kinds of carriers

v) Risk threshold: In the supply chain network, each enterprise itself has a certain tolerance for risk. The threshold is a value that determines whether the node enterprises can normally transmit material/product, information and finance. When this limit is reached, risk will be transmitted form upstream business entity to downstream.

\section{Generation Process}

\subsection{The Selection of Local World}

In the directed supply chain network, the new added node will select cooperation partners and then establish relation with them, so its local world is all the associated nodes.

According to the categories and level of nodes, there are three ways to select:

a) When the new added node is supplier, it will establish business relation with other suppliers or sellers, so all the suppliers and sellers are selected as its local world.

b) When the new added node is seller, it will establish business relation with suppliers; customers or other sellers, so all nodes of the network are selected as its local world.

c) When the new added node is customer, it will establish business relation with sellers, so all the sellers are selected as its local world.

\subsection{Connection Mechanism}

At each step of the evolution, generate a number e of edges and connect them together by adding new node, or, to put it another way, a new added node can establish business relation with $\mathrm{k}$ nodes by immanent probability. In this paper, one must take into consider that the node's risk value may affect its probability proportional of node connection. The 
connection probability proportional $p_{\text {connection }}(k)$ is defined by

$$
p_{\text {connection }}(k)=\frac{\prod_{l \in \mathrm{A}}^{\mathrm{m}} \mathrm{r}(1)-r(k)}{(m-1) * \prod_{\mathrm{l} \in \mathrm{A}}^{\mathrm{m}} \mathrm{r}(1)}
$$

Here, $\mathrm{m}$ is the total number of risk nodes, $r(k)$ is the risk value of new node $\mathrm{k}, r(l)$ is the risk value of its associated node. A is the ultimate supply chain network.

According to different categories and level of nodes, we have the following algorithm:

(i) As a new supplier, it connects to existed node within its local world. In supply chain, each new node will bring $e_{1}$ edges which include $e_{1 \text { in }}$ incoming edges and $e_{1 \text { out }}$ outgoing edges. Goods or raw materials are always flowing from lower level node and finally arriving the higher one. Because the lowest-level suppliers have no supply channel, so $e_{1 \text { in }}$ can be 0 , but $e_{1 \text { out }}$ not.

(ii) As a new seller, it connects to existed node within its local world. In supply chain, each new node will bring $e_{2}$ edges which include $e_{2 \text { in }}$ incoming edges and $e_{2 o u t}$ outgoing edges. Goods or raw materials are always flowing from lower level node and finally arriving the higher one. Because each seller must has supply channel and sale channel, so $e_{2_{\text {in }}}$ and $e_{2 \text { out }}$ can not be 0 .

(iii) As a new customer, it connects to existed node within its local world. In supply chain, each new node will bring $e_{3}$ edges which include $e_{3 \text { in }}$ incoming edges and $e_{3 o u t}$ outgoing edges. Goods or raw materials are always flowing from lower level node and finally arriving the higher one. Because each seller must have supply channel and sale channel, so $e_{3 o u t}$ can be 0 , but $e_{3 \text { in }}$ not.

\subsection{Risk Allocation Process}

In this paper, we assume that the risk value of the associated nodes in the supply chain network model is distributed according to a given risk value $r(k)$. However, each assigned risk value is random, so we must calculate the probability proportional $p(i)$. The distribution probability proportional $p_{\text {distributi on }}(i)$ is defined by:

$$
p_{\text {distribution }}(i)=\frac{2 \arctan \left|\frac{1}{r(k)-40}\right|}{\pi} * \frac{\prod_{l \in A}^{n} r(l)-r(i)}{(n-1) * \prod_{l \in A}^{n} r(l)}
$$

Here, $r(l)$ is all risk value of its associated risk nodes, $r(i)$ is the risk value of its associated risk nodes $\mathrm{i}, \mathrm{n}$ is the total number of its associated risk nodes.
Then, we immediately have the actual risk value $r^{\prime}(i)$

$$
r^{\prime}(i)=r(i)+r(i) * p_{\text {distribution }}(i)
$$

Here, the risk value is cumulated process. We must calculate all risk value of added node which associated with node $i$. The final cumulated value is the actually risk value of nodes $\mathrm{i}$.

\subsection{Molding Process}

The supply chain network model based on risk connection is generated by the following algorithm:

1) Start with a small number $\mathrm{m} 0$ of nodes and confirm the risk of node, the edges, direction and its risk spread probability.

2) A new coming node enters into the supply chain network, select its local world from the existing network.

3 ) The new coming nodes connected with existed nodes by probability $p_{\text {connection }}(k)$.

4) The risk value of associated nodes increase by probability $p_{\text {distributi on }}(i)$

5) Modify the risk of associated node by using formula (1) and (2).

6) Repeat 2), 3), 4), 5) until the number of nodes in the network reaches to $\mathrm{N}$.

\section{Conclusions}

Networks are not only relevant for many current issues but have found application in a variety of supply chain systems. In this paper, we present a weighted supply chain complex network model based on risk. The nodes of model are classified into three types, including supplier, seller and customer. For different categories and level of node, its local world and connection mechanism are different. Furthermore, the new added node is possible to affect its associated nodes.

\section{Acknowledgments}

This paper is supported by Humanity and Social Science Youth foundation of Ministry of Education under Grant Nos. 10YJC630271.

\section{References}

[1] Douglas M Lambert, Martha C Cooper. Issues in Supply Chain Management [J]. Industrial Marketing Management. 2000, 29(1): 65-83.

[2] Hokey Mina, Gengui Zhoub. Supply chain modeling: past, present and future $[\mathrm{J}]$. Computers \& Industrial Engineering. 2002, 43(1): 231-249.

[3] Nakamaru M, Levin S A. Spread of two linked social norms on complex interaction networks [J]. Journal of Theoretical Biology. 2004, 230(1): 57-64. 
[4] Lei W, Mingfang G, Lijun W. The Directed Complex Network Application in the Supply Chain[C]//Digital Manufacturing and Automation (ICDMA), 2012 Third International Conference on. IEEE, 2012: 911-914.

[5] Christopher M, Peck H. Building the resilient supply chain [J]. International Journal of Logistics Management, 2004, 15(2): $1-14$

[6] Pinior B, Konschake M, Platz U, et al. The trade network in the dairy industry and its implication for the spread of contamination [J]. Journal of dairy science, 2012.

[7] Kathrin Büttner,Joachim Krieter, Arne Traulsen,Imke Traulsen. Static network analysis of a poultry supply chain in Northern Germany-Characterisation of the potential spread of infectious diseases via animal movements [J]. 2013, 110(3-4): 418-428.

[8] Van Steenwinkel S, Ribbens S, Ducheyne E, et al. Assessing biosecurity practices, movements and densities of poultry sites across Belgium, resulting in different farm risk-groups for infectious disease introduction and spread [J]. Preventive veterinary medicine, 2011, 98(4): 259-270.
[9] Monique A. van der Gaaga Fred Vosa et al. A state-transition simulation model for the spread of Salmonella in the pork supply chain [J]. European Journal of Operational Research, 156(2004). pp. 782-798.

[10] Paul M, Baritaux V, Wongnarkpet S, et al. Practices associated with Highly Pathogenic Avian Influenza spread in traditional poultry marketing chains: Social and economic perspectives[J]. Acta tropica, 126(2013). pp. 43-53.

[11] Fabrizio Natalea et al.Network analysis of Italian cattle trade patterns and evaluation of risks for potential disease spread [J]. Preventive Veterinary Medicine, 4(2009). pp. 341-350

[12] Monique A. van der Gaaga Fred Vosa et al. A state-transition simulation model for the spread of Salmonella in the pork supply chain [J]. European Journal of Operational Research, 156(2004). pp. 782-798.

[13] Kathrin Büttner,Joachim Krieter, Arne Traulsen,Imke Traulsen. Static network analysis of a poultry supply chain in Northern Germany - Characterisation of the potential spread of infectious diseases via animal movements [J]. 2013, 110(3-4): 418-428. 